11. S. Straszewicz, Über die Zerschneidung der Ebene durch abgeschlossene Mengen, Fund. Math. vol. 7 (1925) pp. 159-187.

12. - Über eine Verallgemeinerung des Jordan'schen Kurvensatzes, Fund. Math. vol. 4 (1923) pp. 128-135.

The UnIversity OF TeXas

\title{
ON ISOMETRIES OF SQUARE SETS
}

PAUL J. KELLY

1. Introduction. It is not fully known under what conditions the isometry of two square, metric sets, say $E^{2}$ and $F^{2}$, implies the isometry of $E$ and $F$. Using the notion of order two self-isometries, this paper gives conditions sufficient to imply $E$ isometric to $F$ when $E^{2}$ and $F^{2}$ are finite and are metrized under any one of a fairly extensive class of functions. The basic ideas are first applied to non-square sets to yield a more general theorem which is then applied to the inverse square problem.

2. Definitions. A set is called metric if to every pair of its elements, $a$ and $b$, there corresponds a real, non-negative number, which is independent of the order of $a$ and $b$, zero if and only if $a$ equals $b$, and which satisfies the triangle law.

Two metric sets are isometric (written " $\equiv "$ ) if there is a one-to-one transformation of one set on the other in which the metric number associated with any pair is the same as that associated with the transformed pair.

A non-identity mapping of a set on itself, which is an isometry, and which leaves each element of the set invariant or else interchanges it with another, is called a self-isometry of order two. Any subset on which the self-isometry is the identity is said to be left pointwise invariant.

Theorem 1. Assume $A \equiv B$ under a mapping $T$, where $A$ and $B$ are finite metric sets. Let $A$ and $B$ have self-isometries of order two under mappings $R$ and $S$ respectively and let $A_{1}$ and $B_{1}$ denote respectively the maximum subsets left pointwise invariant. If $A_{1}$ has no self-isometry of order two, and has at least as many elements as $B_{1}$, then $A_{1} \equiv B_{1}$ and there

Presented to the Society, November 25, 1944, under the title Some properties of a certain interchange type of self-isometry; received by the editors September 23, 1944. 
exists a composition of $R, S, T$ and $T^{-1}$ which maps $A$ isometrically on $B$ and carries $A_{1}$ into $B_{1}$.

Proof. Starting with the set $A_{1}$ the following sequence of sets outlined is obtained by transforming $A_{1}$ by $T$, the set obtained by $S$, this set by $T^{-1}$, and this set by $R$, and so on repeating cyclically the transformations $T, S, T^{-1}, R$.

\section{Column 1}

Column 2

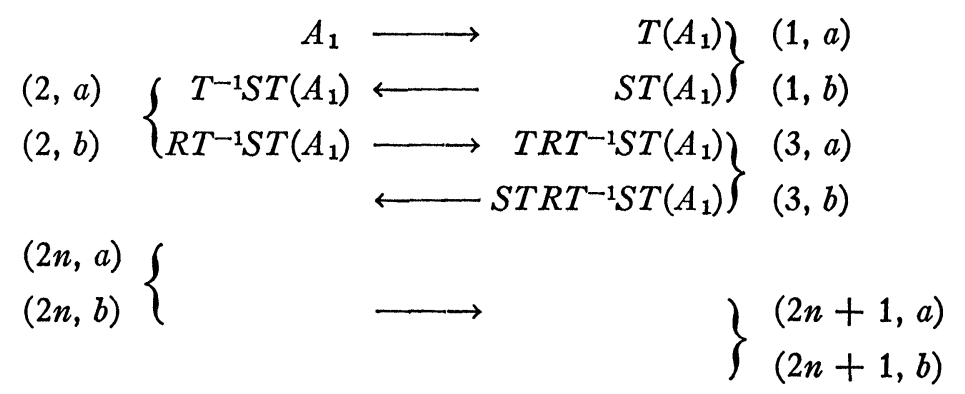

The notation at the side is such that set $(n, x), x=a$ or $b$, is in $B$ if $n$ is odd and in $A$ if $n$ is even. From the construction and the nature of $R$ and $S$, the following relations are easily verified: $R(2 n, a)=(2 n, b)$, $R(2 n, b)=(2 n, a), S(2 n+1, a)=(2 n+1, b), S(2 n+1, b)=(2 n+1, a)$, $T(2 n, b)=(2 n+1, a), T^{-1}(2 n+1, b)=(2 n+2, a)$.

(1) Assume no set in column 2 is the set $B_{1}$.

(2) Since all sets in both columns are isometric to $A_{1}$, isometry being transitive, and since $A_{1}$ has as many elements as $B_{1},(1)$ implies that no set in column 2 is a subset of $B_{1}$.

(3) For any $n, x=a$ or $b, S(2 n+1, x) \neq(2 n+1, x)$. Since $S$ is the identity mapping only on $B_{1}$ and since, from (1) and (2), $(2 n+1, x)$ is not $B_{1}$ or a subset of it, $S(2 n+1, x)=(2 n+1, x)$ would mean that $(2 n+1, x)$ had a self-isometry of order two. This, together with $A_{1} \equiv(2 n+1, x)$, would imply $A_{1}$ had a self-isometry of order two, contradicting the given conditions.

(4) For any $n$, no two sets of column 1 up to and including $(2 n, a)$ are identical. The proof is by induction.

(4.1) Statement (4) holds for $n=1$, since $A_{1}=(2, a)$ would give $T\left(A_{1}\right)=T(2, a)$ or $(1, a)=(1, b)$, contradicting (3).

(4.2) Assume (4) holds for $n=k$.

(4.3) Since $R$ is the identity only on $A_{1}$ and since $(2 k, a)$ is not a subset of $A_{1}$, being isometric to it, and is not equal to $A_{1}$, from (4.2), then $R(2 k, a)=(2 k, a)$ would imply that $(2 k, a)$ had a self-isometry of 
order two, and hence that $A_{1}$ did also. Therefore $R(2 k, a) \neq(2 k, a)$, that is $(2 k, b) \neq(2 k, a)$. This, in turn, implies $(2 k, b) \neq A_{1}$.

(4.4) For $i<k, x=a$ or $b,(2 k, b) \neq(2 i, x)$. From $(2 k, b)=(2 i, x)$ would follow $R(2 k, b)=R(2 i, x)$, that is $(2 k, a)=R(2 i, x)$, which for $i<k$ would contradict (4.2).

(4.5) From (4.2), (4.3), and (4.4) no two sets of column 1 up to and including $(2 k, b)$ are identical. This, with the one-to-oneness of $T$, implies that no two sets of column 2 up to and including $(2 k+1, a)$ are identical.

(4.6) From (3), $(2 k+1, b) \neq(2 k+1, a)$.

(4.7) For $i<k, x=a$ or $b,(2 k+1, b) \neq(2 i+1, x)$. For, from $(2 k+1, b)=(2 i+1, x)$ would follow $S(2 k+1, b)=S(2 i+1, x)$, that is $(2 k+1, a)=S(2 i+1, x)$, which for $i<k$ would contradict (4.5).

(4.8) From (4.6) and (4.7) no two sets of column 2 up to and including $(2 k+1, b)$ are identical. This, with the one-to-oneness of $T^{-1}$, implies that no two sets of column 1 up to and including $(2(k+1), a)$ are identical, and completes the induction establishing (4).

(5) Since (4) implies the existence of an unlimited number of distinct subsets in the finite set $A$, it is clearly a contradiction reached through assuming (1). Therefore (1) is false and $B_{1}$ must occur in column 2 and be isometric to $A_{1}$. The remainder of the theorem follows from the fact that the sequence of sets can be started with $A$ rather than $A_{1}$.

If $A$ and $B$ are the same set and $T$ is replaced by the identity, Theorem 1 reduces to the following result:

TheOREM 2. Let $A$ be a finite metric set and let $A_{1}$ and $B_{1}$ be the maximum subsets left pointwise invariant under two distinct self-isometries, $R$ and $S$, of order two. If $A_{1}$ has no self-isometry of order two and has at least as many elements as $B_{1}$, then $A_{1} \equiv B_{1}$ and there is a composition of $R$ and $S$ which maps $A$ isometrically on itself and carries $A_{1}$ into $B_{1}$.

3. Definitions concerning square sets. Let $E$ be a finite metric set with elements $x_{1}, x_{2}, \cdots, x_{n}$ and metric $\rho_{E}$. By $E^{2}$ is meant the set of couples obtained from the cartesian product of $E$ with itself.

In $E^{2}$ the subset of couples $\left(x_{i}, x_{i}\right), i=1,2, \cdots, n$, is called the diagonal set.

The reflection mapping, $R$, of $E^{2}$ on itself is defined by $R\left(x_{i}, x_{j}\right)$ $=\left(x_{j}, x_{i}\right)$.

If a metric $\rho_{E^{2}}$ is defined on the elements of $E^{2}$ it is called a metric of class $\alpha$ if, in addition to making $E^{2}$ a metric set, it has the following properties : 
(1) For any two points of $E^{2}, P_{1}:\left(x_{i}, x_{j}\right), P_{2}:\left(x_{k}, x_{l}\right), \rho_{E^{2}}\left(P_{1}, P_{2}\right)$ $=f\left(X_{1}, X_{2}\right)$ where $X_{1}=\rho_{E}\left(x_{i}, x_{k}\right), X_{2}=\rho_{E}\left(x_{j}, x_{l}\right)$.

(2) $f\left(X_{1}, X_{2}\right)=f\left(X_{2}, X_{1}\right)$.

(3) There exists a constant $M$ associated with $f$, such that whenever $X_{1}=X_{2}$, then $f\left(X_{1}, X_{2}\right)=M X_{1}$.

THEOREM 3. Let $E$ and $F$ be finite metric sets, and let $E^{2}$ and $F^{2}$ be metrized under the same class $\alpha$ metric. If either the diagonal set of $E^{2}$ or that of $F^{2}$ has no self-isometry of order two, then $E^{2} \equiv F^{2}$ implies $E \equiv F$.

PRoof. Let $R$ and $S$ denote respectively the reflection mappings of $E^{2}$ and $F^{2}$ on themselves. From the definition of reflection and from property 2 of a class $\alpha$ metric, the mappings $R$ and $S$ establish selfisometries of order two in which the diagonal sets alone are left pointwise invariant. The two diagonal sets also have the same number of elements because $E^{2} \equiv F^{2}$. From Theorem 1 , then, with $E^{2}$ and $F^{2}$ playing the roles of $A$ and $B$, and with the diagonal sets as $A_{1}$ and $B_{1}$, it follows that the diagonal set of $E^{2}$ is isometric to that of $F^{2}$. This isometry together with property 3 of a class $\alpha$ metric implies $E \equiv F$.

UNIVERSITY OF WISCONSIN 